\title{
The Efficacy of Combined Plasma Gel and Fractional CO2 Laser in Treatment of Atrophic Acne Scars H.H.Sabry ${ }^{1}$, R.M.Salem ${ }^{2}$ and M.M.Mohamed ${ }^{1}$ \\ ${ }^{1}$ Dermatology, Venereology and Andrology Dept., Faculty of Medicine, Benha Univ., Benha, Egypt ${ }^{2}$ Dermatology, Venereology and Andrology Dept., Faculty of Medicine, Benha Univ., Benha, Egypt E-Mail: mai.mustafa88@gmail.com
}

\begin{abstract}
Atrophic post acne scars have a profound psychosocial impact on patients of all ages. The dermatologists usually face the dilemma of choosing the most effective or choosing the safest modality of treatment. The fractional $\mathrm{CO} 2$ laser (FCL) resurfacing is the gold standard for treating atrophic scars but is associated with prolonged recovery and many side effects. To address these limitations, newer combined therapies have emerged. The current work aimed to evaluate the safety and efficacy of FCL combined with topical application of plasma gel in the treatment of atrophic acne scars.Twenty patients with facial atrophic post acne scars were enrolled in this study. All of them completed the study. All patients underwent 4 treatment sessions at 4 - weeks intervals. The evaluation was applied by the investigator at the baseline, and one month after final session. Then, the percentage of improvement was calculated. Patients were with Fitzpatrick skin types II-V. They were 8 males and 12 females. According to the qualitative global scarring grading system (GSGS), patients with grade 4 acne scars comprised $50 \%$ of the study population, followed by $10 \%$ with grade 2 and $40 \%$ with grade 3. GSGS scores were decreased from $3.10 \pm 0.99$ to $2.00 \pm 0.47$ after 1 month from the last treatment session with $P$ - value $<0.001$. Reported side effects were mild and transient.The combination of plasma gel and FCL resurfacing was noticeably outstanding in their efficacy and safety.
\end{abstract}

Keywords: Plasma gel, PRP-gel, Fractional laser, Atrophic acne scar.

\section{Introduction}

Atrophic post acne scarring is often a poor and permanent sequel of acne vulgaris. Scarring may occur early in acne and may affect 95 percent of patients with acne, relating to both its severity and delay before treatment. It has high prevalence, significant impact on quality of life, and therapeutic challenge for dermatologists [1]. The treatment of atrophic post acne scars varies depending on the extent and depth of acne scars and the limitations of the treatment modalities in their ability to improve scars and in their safety. Plenty of options are available for the treatment of acne scarring, including chemical peeling, dermabrasion, ablative laser treatment, punch techniques, fat transplantation, dermal fillers, microneedling, subcision, and combined Platelet rich plasma (PRP) therapies. There are also promising procedures, such as stem cell therapy. The modalities used to treat post acne scars have shown efficacy, but variable results on different patients and problematic side effects have restricted their application [2].

Fractional resurfacing is a laser treatment modality that Manstein and colleagues introduced in 2004, to bridge the gap between ablative and nonablative resurfacing 3 . Fractional resurfacing lays down a matrix of energy beams to form an array of microscopic thermal wounds (microscopic treatment zones) to stimulate a therapeutic response in the dermis [4]. The reservoir of spared skin allows rapid repair of laser-induced injury [5].

Although fractional resurfacing does not produce results comparable with those of full ablative laser skin resurfacing, it has quickly become much more popular than the latter because the side effects, risks, and downtime of treatment are limited and improvement is acceptable [6].
The use of autologous blood derived products in the field of cosmetic dermatology and plastic surgery has been discussed at length. In particular, (plasma gel), has gained popularity in treatment of chronic wounds [7]. The advent of plasma gel therapy within the past few years has emerged and replaced dermal fillers and Platelet Rich Plasma (PRP) in their applications [8].

\section{Material and methods}

This study was conducted as a prospective clinical trial that involved 20 patients above the age of 18 years recruited from Dermatology outpatient clinic, Benha University Hospital. In the present study 8 males and 12 females were enrolled. All details of the study were discussed with the patients and informed consent was obtained from each patient before enrolment in the study. The protocol was revised and approved by the Ethics Committee of Human Research., Faculty of Medicine, Benha University.

Patients with any of the following conditions were excluded from the study: Bleeding tendency e.g. Critical thrombocytopenia (low platelet count), anticoagulation therapy, the use of oral retinoid drugs within the last 6 months, personal or family history of keloid scarring, collagen vascular disease, photosensitivity or current use of photosensitive drugs, history of post inflammatory hyperpigmentation, presence of acute or chronic active skin infections or Sepsis.

\subsection{Baseline evaluation}

All Patients were subjected to the following:

\subsubsection{History taking}

Patients' demographic data were recorded: age, sex, and occupation. A detailed history was taken from patients regarding treatment of medical relevance e.g. 
topical and systemic retinoids, anticoagulants, systemic steroids and relevant infections e.g. Herpes simplex virus infection. Patients were asked about medical problems as diabetes, liver disease, or bleeding tendency. Also, patients were asked about past cosmetic procedures as laser resurfacing, chemical peeling, dermabrasion or subscision.

\subsubsection{Clinical examination}

Full dermatological examination was done to exclude the presence of active acne lesions, current Table (1) Qualitative global scarring grading system. cutaneous infections, inflammatory lesions or tumors. Also, detection of the skin phenotype was carried out according to the Fitzpatrick classification.

Post acne scars were assessed and graded by the qualitative GSGS. This system provides a simple grading of a patient with post acne scarring and allow the rational description of that patient. It subdivides acne scars into grades 1-4 based on scar severity (macular, mild, moderate, and severe, respectively) (Table 1) [9].

\begin{tabular}{lcr}
\hline Grades & $\begin{array}{c}\text { Level of } \\
\text { disease }\end{array}$ & Clinical features \\
\hline 1 & Macular & $\begin{array}{r}\text { These scars can be erythematous, hyper- or hypopigmented flat marks. They do not } \\
\text { represent a problem of contour like other scar grades but of colour. }\end{array}$ \\
Mild & $\begin{array}{r}\text { Mild atrophy or hypertrophy scars that may not be obvious at social distances of } 50 \mathrm{~cm} \\
\text { or greater and may be covered adequately by makeup or the normal shadow of } \\
\text { shaved beard hair in men or normal body hair if extrafacial. }\end{array}$ \\
Moderate & $\begin{array}{r}\text { Moderate atrophic or hypertrophic scarring that is obvious at social distances of } 50 \mathrm{~cm} \\
\text { or greater and is not covered easily by makeup or the normal shadow of shaved beard } \\
\text { hair in men or body hair if extrafacial, but is still able to be flattened by manual } \\
\text { stretching of the skin (if atrophic). }\end{array}$ \\
& $\begin{array}{r}\text { Severe atrophic or hypertrophic scarring that is evident at social distances greater than } \\
50 \text { cm and is not covered easily by makeup or the normal shadow of shaved beard hair } \\
\text { in men or body hair if extrafacial and is not able to be flattened by manual stretching of } \\
\text { the skin. }\end{array}$ \\
\hline
\end{tabular}

\subsubsection{Photographic documentation}

All photographs were taken for the face using digital camera (Nikon D5200 24.1 MP CMOS Digital SLR camera, Thailand) using fixed settings, lighting and patient positioning (right and left profile views of the face) for standardization. The photos were taken for included patients at baseline visit and 1 month after the last treatment session.

\subsection{Treatment protocol}

All patients underwent 4 treatment sessions at $4-$ weeks intervals. Patients were told to stop any topical treatment they were used to apply it for acne or scars at least 2 weeks before the procedure. No other cosmetic procedures were allowed at the intervals between the sessions and during the period of follow up.

At first, areas to be treated were cleansed with mild cleanser. Thereafter, local anesthetic cream Pridocaine cream (2.5\% lidocaine and $2.5 \%$ Prilocaine: GLOBAL NAPI) was applied to the area to be treated for 1 hour before the treatment session in order to mitigate the pain during procedure. Then the whole face was cleansed using either a mild cleanser or moist saline-soaked gauzes and dried with sterile gauze.

The areas of atrophic acne scars of all patients were irradiated with FCL (Smartxide DOT, Advanced CO2 Fractional technology, DEKA, Florence, Italy). Treatment settings were adjusted according to each individual case regarding the skin type. After each session, icepacks were applied immediately to the treated area for 5-10 minutes. Then, the patients underwent topical application of cooled plasma gel to the treated areas. The plasma gel was applied in 2 coats, 15 minutes apart and the patients were advised not to wash the treated area for the next 24 hours. All patients were instructed to avoid direct sun exposure, heat, and friction to the treated areas and to apply sunscreen with a sun protection factor of 50+ daily and a moisturizing cream or post laser soothing agent to promote wound healing and prevent dryness.

\subsection{Plasma gel preparation}

Ten milliliter of venous blood sample was taken from each participant under complete aseptic conditions and then distributed into 4 sterile tubes equipped with anticoagulant sodium citrate solution. Then the tubes were placed in a centrifuge machine to spin at $1500 \mathrm{rpm}$, for 10 minutes at room temperature, resulting in the following three layers: the lower layer composed of red blood cells, the middle layer composed of white blood cells (the buffy coat), and the upper layer composed of plasma. The upper plasma and buffy coat layers were aspirated and centrifuged again for another 10 minutes at $3500 \mathrm{rpm}$. After the second spin step, a soft erythrocyte pellet was formed at the bottom of the tube. The PRP was then gently aspirated and placed in sterile $3 \mathrm{ml}$ syringes, and then prepared for activation by calcium chloride with 
proportion of $0.01 \mathrm{~mL}$ per $1 \mathrm{~mL}$ of plasma. This activated PRP was heated in a hot water path at a temperature between 70 and $100^{\circ} \mathrm{C}$ for 3 minutes, and then in a cold path at a temperature between 5 and $0^{\circ} \mathrm{C}$ for another 3 minutes. Finally, liquid plasma was transformed into a viscous gel ready for use.

\subsection{Assessment of efficacy} (GSGS)

The qualitative global scarring grading system

This system was used by the investigator at the baseline, and 1 month after final session. Then, the percentage of improvement was calculated.

Patient satisfaction.

Patients were asked about their level of satisfaction at the visit one month after the last session.

\subsection{Statistical Analysis}

Statistical presentation and analysis of the present study was conducted, using the mean, standard deviation, student t- test, Paired t-test, Chi-square, Linear Correlation Coefficient and analysis of variance [ANOVA] tests by SPSS V20.

\section{Results and discussion}

The current study was carried out on 20 patients presented with different grades of atrophic post acne scarring as follows: 10 patients $(50 \%)$ with grade 4 acne scars, 8 patients (40\%) with grade 3 and 2 patients (10\%) with grade 2 . Their demographic data were summarized in Table (2).

Table (2) Demographic data of the patients.

\begin{tabular}{lccc}
\hline Demographic data & & & \\
\hline Sex & Male & 8 & $40.0 \%$ \\
& Female & 12 & $60.0 \%$ \\
Age & Range & $19-38$ \\
& Mean & $26.600 \pm$ \\
& \pm SD & 6.979 \\
Skin & II & 2 & $10.0 \%$ \\
Type & III & 6 & $30.0 \%$ \\
& IV & 8 & $40.0 \%$ \\
Scar Duration & V & 4 & $20.0 \%$ \\
(Years) & Range & $1-15$ \\
& Mean & $5.500 \pm$ \\
& \pm SD & 4.972 \\
\hline
\end{tabular}

\subsection{Assessment of efficacy}

At the end of treatment, all patients showed statistically significant clinical improvement in the severity of post acne scarring in comparison with that

before treatment sessions as the mean value of their qualitative GSGS scores decreased from $3.10 \pm 0.99$ at baseline visit to $2.00 \pm 0.47$ after 1 month from the last treatment session with $\mathrm{P}$ - value $<0.001$ Fig (1) .
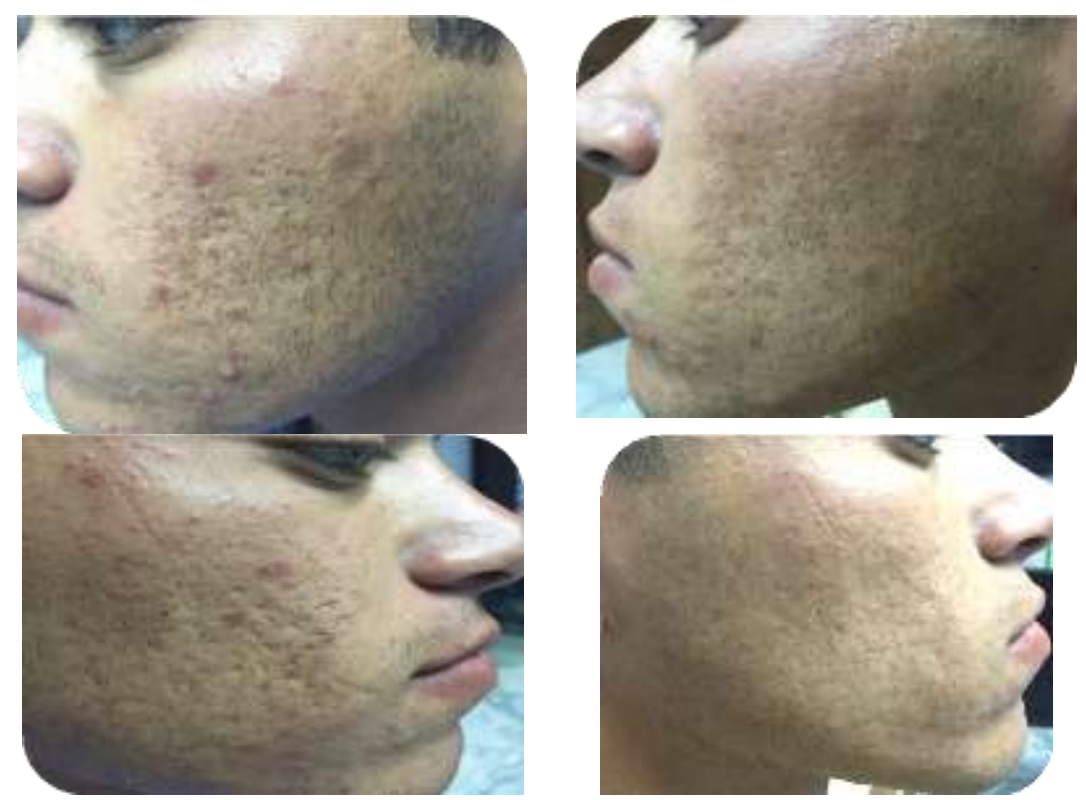

Fig (1) The patient before and I month after treatment. (a) Left side of the face, (b) Right side of the face 
None of the patients showed worsening of the treated area or even no change. Regarding the patient satisfaction level, none reported being dissatisfied and all patients showed varying degrees of satisfaction. Full satisfaction was reported by $60 \%$ of the patients, and $30 \%$ of them reported being markedly satisfied, and $10 \%$ were moderately satisfied with the results at the end of follow up period.

\subsection{Safety assessment:}

The procedure was well tolerated by all the participants. Post procedural side effects were transient and minimal in the form of mild pain, minor burning, erythema, and edema at the treated areas. No events of scarring, dyspigmentation, or infection were reported.

During the past decade, the fractional CO2 laser has been effectively utilized for the treatment of atrophic post-acne scarring [10]. However, this modality has shown some limitations specially when used in patients with darker skin phototypes [11]. Due to a need for safer and more noticeable clinical improvement than the fractional laser monotherapy could provide, the combined treatment modalities were preferred by plenty of studies. Lee et al., (2011) found that the combination of PRP with fractional $\mathrm{CO} 2$ laser was very effective in the treatment of post-acne scarring and it had significantly shortened the post- procedural downtime. [12]

Platelets rich plasma is used by many physicians in an inactivated liquid form that can be easily injected. After contact with the surrounding tissue collagen, platelets were activated [13]. As another option, exogenous activator as calcium chloride, thrombin, or combination of both can be added to form the PRP-gel $[14,15]$.

To the best of our knowledge, this was the first study that evaluated the clinical efficacy and safety of combined plasma gel and fractional $\mathrm{CO} 2$ laser for the treatment of atrophic post-acne scarring.

This improvement and noticeable dampening of side effects found to be consistent with findings in previous studies that used the liquid form of PRP. Kar and Raj conducted a split-face comparative study on 30 patients with Goodman severity 3-4. Patients reported significantly less severity of erythema, edema and pain symptoms on the side treated with combined fractional CO2 laser and topical PRP against the side treated with fractional laser alone[16].

Kim and Gallo evaluated the effect of PRP on recovery after fractional $\mathrm{CO} 2$ laser resurfacing. Across 94 observations, they found a statistically significant improvement in edema and erythema in PRP treated arms. Moreover, patients themselves have noticed a reduction in the post-treatment effects: erythema, edema, pruritus, and discomfort as compared to the saline- treated arms [17].

Min et al., used the immunohistochemistry to confirm that PRP treatment could intensify the fibrogenetic molecules induced by the fractional $\mathrm{CO} 2$ $\beta 1$, TGF- $\beta 3$, collagen 1 , and collagen 3 in acne scar skin tissues after treatment with PRP and fractional $\mathrm{CO} 2$ laser compared with laser treatment alone. PRP treatment also produced increased epidermal growth factor receptor (EGFR) expression and decreased keratin 16 in keratinocyte at 48 hours, strongly suggesting that PRP accelerate epithelization and decrease laser induced skin damage. Additionally, they attributed the soothing effect of PRP to the upregulation of TGF $\beta$ and hepatocyte growth factor (HGF) and associated decreased levels of C- FOS and AP-1 pathway [18].

Jalowiec et al., approved in their study that plasma gel tends to maintain its shape instead of crawling and permeating like a viscous fluid because of the effective cross-linking at the molecular level achieved during the gelification process $[15,19]$.

Considering that acne scar treatment is a lengthy process and requires a combination of procedures, early initiation of treatment could greatly improve the quality of life of patients.

Treatment parameters can vary between the sessions for example, treatment coverage can increase throughout the treatment series. It has been observed that treatments became more tolerable and the recovery became increasingly faster and better throughout the treatment series.

There are numerous advantages to harness the therapeutic and protective effects of plasma gel. First, PG can be easily prepared from peripheral blood samples, which is a relatively noninvasive technique. Second, PG is an autologous material, which minimizes the risk of immune reactions and pathogen transmission compared with non-autologous material. Third, it is economical. But it has the disadvantage of the need for multiple sessions.

The limitations of the present study naturally include: a small number of patients participated in the studied groups and difficult assessment of efficacy on each scar type individually. Another limitation in the methods utilized involves the need for 3- dimensional assessment of the improvement.

\section{Conclusion}

In light of the current study, application of autologous plasma gel after acne scars resurfacing with fractional $\mathrm{Co} 2$ laser is very effective and well tolerated.

\section{References}

[1] M.Boen and C.Jacob, A Review and Update of Treatment Options Using the Acne Scar Classification System. Dermatol Surg; Vol.45(3), PP. 411-422, 2019

[2] M.V. Gozali and B.Zhou, Effective treatments of atrophic acne scars, J Clin Aesthet Dermatol; Vol. 8(5), PP. 33-40, 2015.

[3] D.Manstein, G.S. Herron, R.K. Sink, H .Tanner and R.R. Anderson, Fractional Photothermolysis: A 
New Concept for Cutaneous Remodeling Using Microscopic Patterns of Thermal Injury, Lasers Surg Med, Vol. 34(5), PP. 426-438., 2004.

[4] M .Lapidoth, S .Halachmi, S .Cohen and D.B. Amitai, Fractional $\mathrm{CO} 2$ laser in the treatment of facial scars in children, Lasers Med Sci, Vol. 29(2), PP. 855-857, 2014.

[5] G.H. Park, S.E. Chang, S .Bang, K.H. Won, C.H. Won, M.W. Lee, J.H. Choi and K.C. Moon ,Usefulness of Skin Explants for Histologic Analysis after Fractional Photothermolysis .Ann Dermatol; Vol. 27(3): PP. 283-290, 2015.

[6] M .Naouri, M .Atlan, E .Perrodeau, G. Georgesco, R. Khallouf, L. Martin and L. Machet, High-Resolution Ultrasound Imaging to Demonstrate and Predict Efficacy of Carbon Dioxide Fractional Resurfacing Laser Treatment, Dermatol Surg; Vol. 37(5),PP. 596-603, 2011.

[7] Piccin A, Di Pierro AM, Canzian L, Primerano M, Corvetta D, Negri G, Mazzoleni G et al 'Platelet gel: a new therapeutic tool with great potential. Blood transfusion; 15(4): 333-340, 2017.

[8] A.P. Sclafani and J .Azzi, Platelet Preparations for Use in Facial Rejuvenation and Wound Healing: A Critical Review of Current Literature, Aesthetic Plast Surg, Vol. 39(4): PP. 495-505., , • 10.

[9] G.J. Goodman and J.A. Baron, Postacne scarring: a qualitative global scarring grading system, Dermatol Surg; vol, 32(12), PP.1458-66, 2006.

[10] Y. Xu and Y .Deng, Ablative Fractional CO2 Laser for Facial Atrophic Acne Scars, Facial Plast Surg, Vol. 34(2), PP. 205-219., 2018.

[11] X.H Yuan, S.X Zhong and S.S Li, Comparison study of fractional carbon dioxide laser resurfacing using different fluences and densities for acne scars in Asians: a randomized split-face trial, Dermatol Surg, Vol. 40(5): PP. 545-552, 2014.

[12] J.W. Lee, B.J .Kim, M.N. Kim and S.K. Mun, The Efficacy of Autologous Platelet Rich Plasma Combined with Ablative Carbon Dioxide Fractional
Resurfacing for Acne Scars: A Simultaneous SplitFace Trial, Dermatol Surg; Vol. 37(7), PP. 931938,2011.

[13] D.E .Roberts, A .McNicol and R .Bose, Mechanism of collagen activation in human platelets .

J Biol Chem, Vol. 279(19): PP.19421-19430, 2004.

[14] P .Galindo Moreno, G. Avila Ortiz, L .Torrecillas Martinez, J.E. Fernández Barbero, F. O’Valle and H.L. Wang, Composition of platelet-rich plasma gel: a Western blot analysis, Oral Science Rehabilitation, Vol.2(2), PP.42-,2016.

[15] J.M. Jalowiec, M. D'Este, J.J .Bara, J. Denom, U. Menzel, M. Alini, S .Verrier and M .Herrman ., An In Vitro Investigation of Platelet-Rich Plasma-Gel as a Cell and Growth Factor Delivery Vehicle for Tissue Engineering, Tissue engineering. Part C, Methods; VOL.22(1), PP.49-5, 2016.

[16] B. Kar and C. Raj, Fractional CO2 Laser vs Fractional CO2 with Topical Platelet-rich Plasma in the Treatment of Acne Scars: A Split-face Comparison Trial. J Cutan Aesthet Surg, Vol.10(3), PP.136-144, 2017.

[17] H.Kim, and J. Gallo, Evaluation of the effect of platelet-rich plasma on recovery after ablative fractional photothermolysis. JAMA Facial Plast Surg, Vol.17(2), PP. 97-102, 2015.

[18] S. Min, J.Y. Yoon, S.Y. Park, J. Moon, H.H Kwon and D.H. Suh, Combination of platelet rich plasma in fractional carbon dioxide laser treatment increased clinical efficacy of for acne scar by enhancement of collagen production and modulation of laser-induced inflammation, Lasers Surg Med, Vol.50(4), PP. 302-310, 2018.

[19] K.L. Kroeze, W.J. Jurgens, B.Z. Doulabi, F.J. van Milligen, R.J .Scheper and S. Gibbs, Chemokine-Mediated Migration of Skin-Derived Stem Cells: Predominant Role for CCL5/RANTES. J Invest Dermatol, Vol.129(6), PP.1569-1581, 2009. 